\title{
Effect of Vagotomy on Hyperinsulinemia in Obese Rats with Hypothalamic Lesions
}

\author{
Kazumasa ChIKAMORI, Kazuyo MASUdA, Hidehiko IZUMI, \\ KATSUTOSHI ISAKA AND UICHIRO TEZUKA \\ Department of Internal Medicine, School of Medicine, \\ Tokushima University, Tokushima 770, Japan
}

\begin{abstract}
Synopsis
With the purpose of investigating the pathogenesis of obesity and hyperinsulinemia in rats with hypothalamic lesions (HTL), HTL were made in vagotomized rats, and the development of obesity was serially followed up to 15 weeks as well as the changes of plasma triglyceride and immunoreactive insulin (IRI) levels.

Even in vagotomized rats, obesity developed after HTL and plasma triglyceride and IRI levels increased significantly. However, obesity was slightly less in grade and occurred later as compared with sham-vagotomy-HTL rats. Plasma IRI levels in vagotomized rats significantly correlated with the body weight, Lee's index, the weight of adipose tissue and plasma triglyceride level. Similar results were also obtained in rats with HTL which were pair-fed following vagotomy.

These results suggest that the hyperinsulinemia in obese rats with HTL may be involved not only by hypersecretion of insulin mediated by hypothalamo-vagal nerve system but also by some insulin-antagonistic factors such as increases of adipose tissues and plasma lipids.
\end{abstract}

In order to elucidate the cause of obesity, we have studied the pathogenesis of obesity in rats with hypothalamic lesions (HTL) and the following results have been reported previously. 1. Rats with HTL have a tendency of hyperphagia from the early stage afer HTL and become markedly obese. 2. Fatty acid composition in the adipose tissue shows a decrease of the linoleic: palmitic ratio, and it is mimicked by the administration of insulin to normal rats (Kuwata, 1970). 3. Plasma immunoreactive insulin (IRI) level is markedly elevated, and the pancreatic islets are enlarged after HTL (Chikamori et al., 1973). 4. These alterations observed in the rats with HTL are not influenced by either restriction of food intake (Suzuki, 1970) or

Received for publication October 18, 1976. hypophysectomy (Hamajo, 1974). 5. Plasma free fatty acid (FFA) and triglyceride levels are elevated in the rats with HTL and the plasma levels of triglyceride and IRI are correlated with the body weight, grade of obesity and the weight of epididymal fat (Chikamori et al., 1973). These results indicate that hyperinsulinemia plays a significant role in the development of obesity in rats with HTL.

The hyperinsulinemia in rats with HTL has been attributed to hyperphagia due to HTL (Hales and Kennedy, 1964), increases in insulin antagonists accompanying obesity (Salans et al., 1968), and insulin hypersecretion from the pancreas mediated by the vagal nerve system (Frohman and Bernerdis, 1971), but exact mechanism has been still unknown. Therefore, the present experiments were designed to study the effect of vago- 
tomy on the development of obesity and hyperinsulinemia in rats with HTL.

\section{Materials and Methods}

\section{Animals and experimental procedures}

Wistar strain male rats weighing about $200 \mathrm{~g}$ were used. Truncal subdiaphragmatic vagotomies were performed according to the method of Snowdon and Epstein (1970) (vagotomy rats).

One week after vagotomy, bilateral electric lesions were made in the area of the ventromedial hypothalamus (HTL rats) by the method described previously (Chikamori, 1969), and sham-operated rats were used as controls (sham-HTL rats). All animals were kept in separate cages and fed ad libitum, but some groups of rats were made pair-feeding to check the effect of food intake. Body weight and food intake were measured twice a week. The degree of obesity was estimated by body weight, body type and Lee's index (1929), and the weight of the epididymal and body fat was also measured. These data in the vagotomy groups were compared with those in the sham-vagotomy groups which were performed in another experiment.

\section{Biochemical analysis}

Before, and 2 days, 1, 4, 10 and 15 weeks after HTL, rats were made to fast for $4 \mathrm{hr}$ and blood samples were collected from the femoral vein under hexobarbital anesthesia. Blood sugar was determined by glucose-oxidase method. Plasma FFA was assayed by Itaya-Ui's method (1965). Plasma triglyceride was determined by acetyl-acetone method (Fletcher, 1968). Plasma IRI level was measured using a commercial insulin radioimmunoassay kit (Dainabot RI Labs.), and expressed as $\mu \mathrm{U} / \mathrm{m} l$ of plasma in terms of the porcine insulin standard. Rat plasma containing endogenous insulin produced an inhibition curve parallel to that obtained with the porcine insulin standard as shown in Figure 1.

\section{Histological investigations}

At the end of the experiments, the brains of the rats were removed and frontal sections were obtained. Hematoxylin-eosin or Nissl stain was performed in order to verify the lesion site histologically. When the site of lesions was not correct, these rats were excluded from the experiment. Furthermore, the evidence of vagotomy was confirmed histologically.

\section{Results}

\section{Body weight, food intake and grade of obesity}

The body weight and food intake of the vagotomized rats with or without HTL were measured during the experiment (Fig. 2a), and compared with those of the shamvagotomy rats obtained in another experiment (Fig. 2b). The vagotomy-HTL rats showed a marked increase in food intake and body weitht as compared with the vagotomy-sham-HTL rats, although their increases were smaller in grade and occurred later as compared with sham-vagotomyHTL rats.

Pair-feeding was made to some rats of both groups, then the body weight was almost identical between vagotomy-HTL rats and vagotomy-sham-HTL rats at respective stages. Fifteen weeks after HTL, however, Lee's index and epididymal and body fat were significantly higher in the vagotomy-HTL rats than in the vagotomysham-HTL rats (Table 1). This indicated that obesity was developed even in vagotomy rats followed by HTL.

\section{Blood sugar, plasma FFA and triglyceride levels}

The changes in blood sugar, plasma FFA and triglyceride levels in the vagotomized rats with or without HTL are

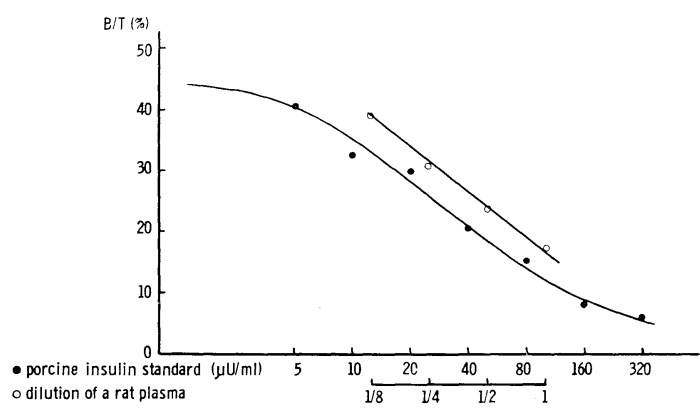

Fig. 1. Inhibition curves in radioimmunoassay with porcine insulin standard and plasma from a rat. 
shown in Figure $3 \mathrm{a}$ and compared with those of the sham-vagotomy rats (Fig. 3b). There were no significant changes in blood glucose levels between HTL and shamHTL groups at their respective stages. Plasma FFA levels tended to be higher in the vagotomy-HTL rats than in the vagotomysham-HTL rats. Plasma triglyceride levels began to rise from 4 weeks after HTL in vagotomy-HTL rats and were significant1y higher than those in the vagotomy-shamHTL rats at the tenth week. But the elevation was not so marked as in the sham-vagomy-HTL rats.

When pair-feeding was made after vagotomy, plasma FFA and triglyceride levels were elevated in vagotomy-HTL rats in comparison with vagotomy-sham-HTL rats, although the difference between two groups was further diminished (Table 1).

\section{Plasma IRI levels}

As seen in Figure 3, plasma IRI levels in vagotomy-HTL rats and vagotomy-sham- (a) vagotomy
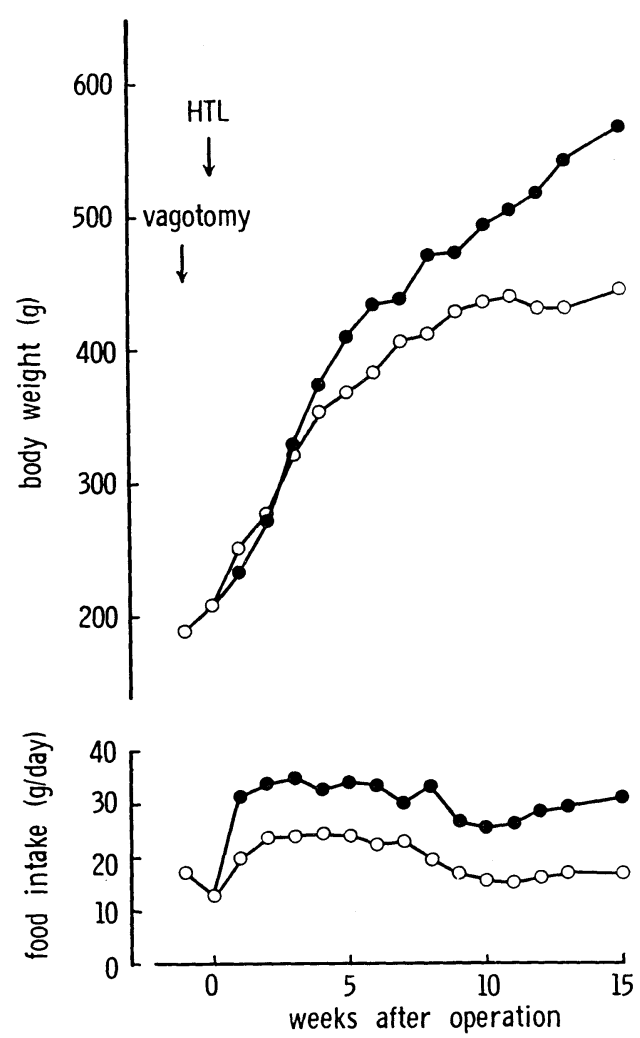

$\multimap$ vagotomy-sham-HTL (5)

$\longrightarrow$ vagotomy-HTL (5) (b) sham-vagotomy.
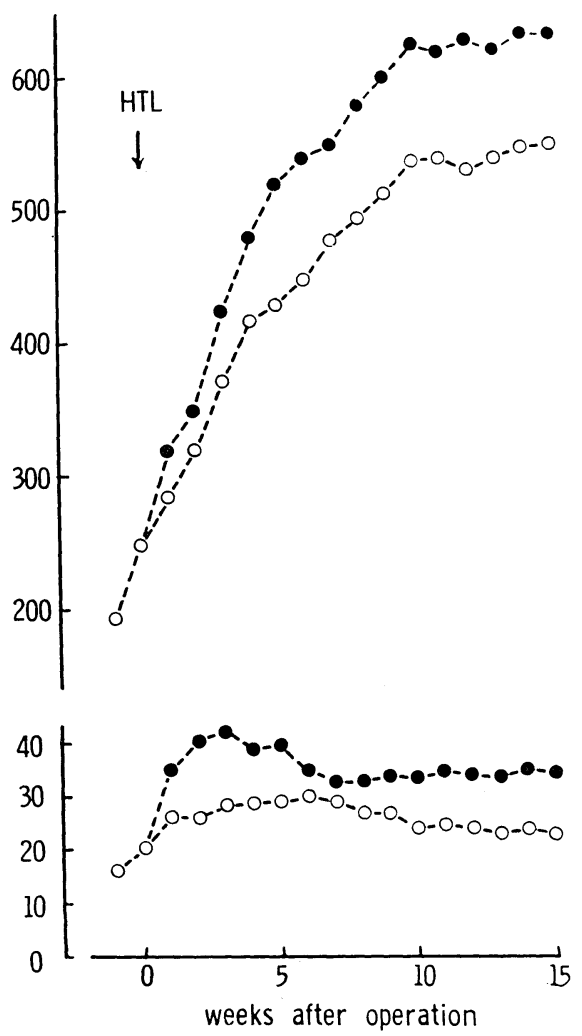

0---o sham-vagotomy-sham-HTL (6)

---• sham-vagotomy-HTL (6)

Fig. 2. Weight gain and food intake of vagotomized or sham-vagotomized rats with or without hypothalamic lesions. Numbers in parentheses represent the number of rats. 
(a) vagotomy
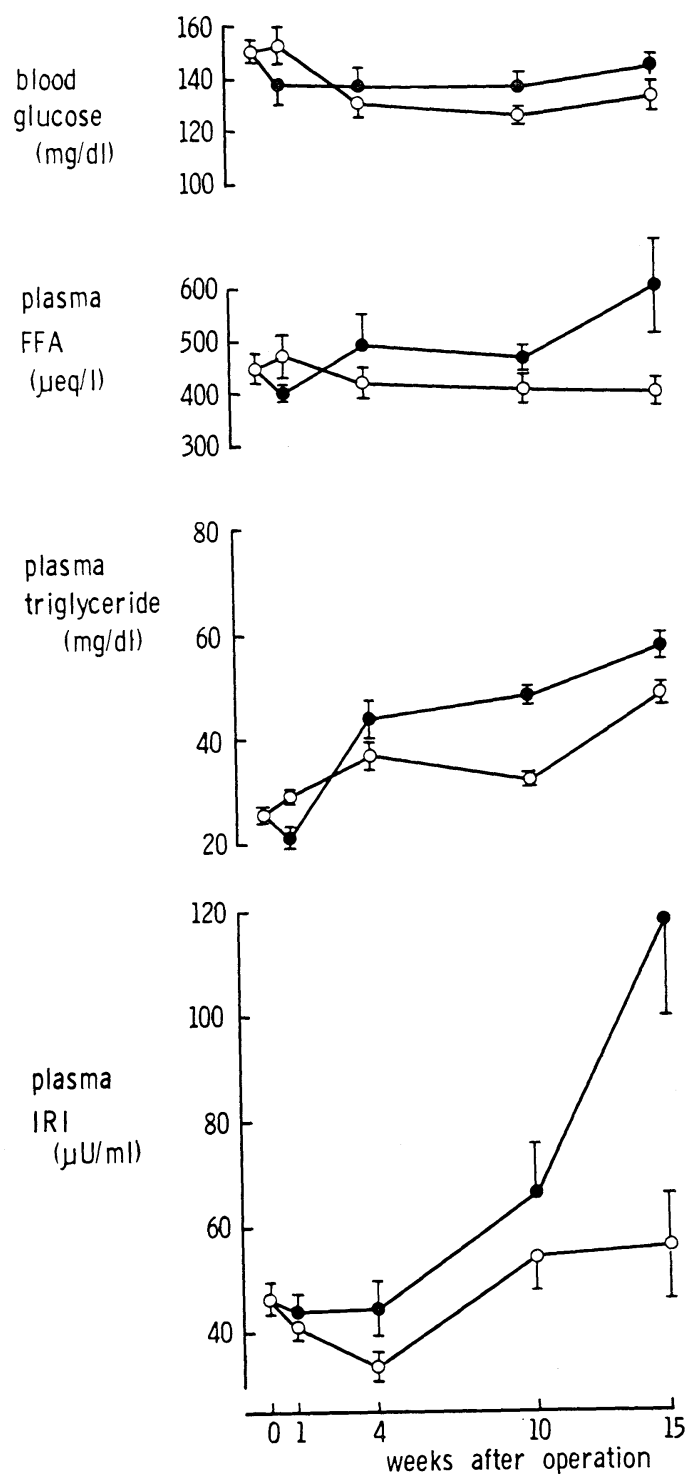

(b) sham-vagotomy
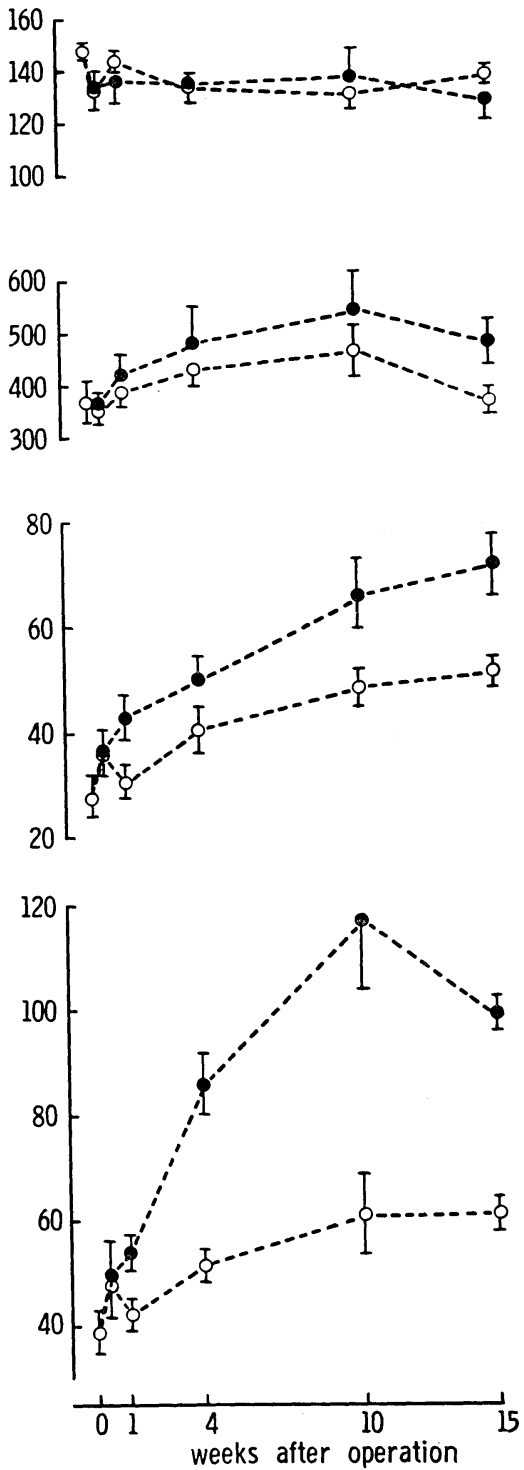

$\multimap$ vagotomy-sham-HTL (5)

$\longrightarrow$ vagotomy-HTL (5) 0---o sham-vagotomy-sham-HTL (6)

---- sham-vagotomy-HTL (6)
I mean \pm SE

Fig. 3. Blood glucose, plasma FFA, triglyceride and IRI levels in vagotomized or sham-vagotomized rats with or without hypothalamic lesions. Numbers in parentheses represent the number of rats. 
Table 1. Various experimental data and their statistical comparisons in pairfed vagotomized rats with or without hypothalamic lesions (15th week after hypothalamic lesions).

\begin{tabular}{lccc}
\hline & Vagotomy-sham-HTL $(6)$ & Vagotomy-HTL $(5)$ & P \\
\hline Initial boby weight $(\mathrm{g})$ & $240.7 \pm 9.2$ & $256.0 \pm 4.6$ & NS \\
Final body weight $(\mathrm{g})$ & $473.7 \pm 10.4$ & $481.6 \pm 7.5$ & NS \\
Weight gain $(\mathrm{g})$ & $262.7 \pm 11.1$ & $280.0 \pm 6.4$ & $\mathrm{NS}$ \\
Naso-anal length $(\mathrm{cm})$ & $25.1 \pm 0.2$ & $24.5 \pm 0.1$ & $\mathrm{NS}$ \\
Lee's index & $0.309 \pm 0.001$ & $0.321 \pm 0.002$ & 0.001 \\
Wt. of epid. fat pad $(\mathrm{g})$ & $7.2 \pm 0.2$ & $11.0 \pm 1.3$ & 0.02 \\
Body fat $(\%)$ & $26.7 \pm 1.0$ & $45.0 \pm 2.7$ & 0.001 \\
Blood glucose $(\mathrm{mg} / \mathrm{d} l)$ & $151.0 \pm 8.9$ & $149.6 \pm 9.0$ & NS \\
Plasma FFA $(\mu \mathrm{eq} / \mathrm{L})$ & $485.8 \pm 93.4$ & $598.4 \pm 65.2$ & NS \\
Plasma triglyceride $(\mathrm{mg} / \mathrm{d} l)$ & $45.4 \pm 16.0$ & $84.6 \pm 12.1$ & 0.05 \\
Plasma IRI $(\mu \mathrm{U} / \mathrm{m} l)$ & $52.2 \pm 4.9$ & $78.0 \pm 7.1$ & 0.05 \\
\hline
\end{tabular}

Mean value \pm S.E. ( ): Number of rats.

HTL rats did not show any significant difference until 10 weeks after HTL. At the fifteenth week, however, the plasma IRI level in the vagotomy-HTL rats was $118.6 \pm$ $18.4 \mu \mathrm{U} / \mathrm{m} l$ in contrast to $56.0 \pm 10.2 \mu \mathrm{U} /$ $\mathrm{m} l$ in the vagotomy-sham-HTL rats. The plasma IRI level was elevated significantly, though it occurred later in comparison with the sham-vagotomy-HTL rats.
By the pair-feeding after vagotomy, the difference of plasma IRI levels between two groups were reduced, but plasma IRI levels were significatly elevated in vagotomyHTL rats 15 weeks after HTL as compared with those in vagotomy-sham-HTL rats (Table 1).

The result of the intravenous glucose tolerance test in pair-fed vagotomized rats 15
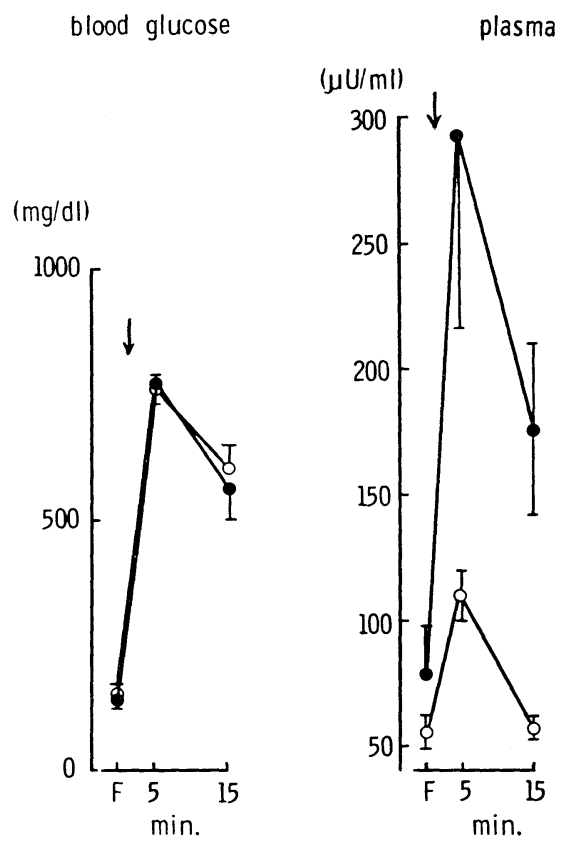

plasma FFA
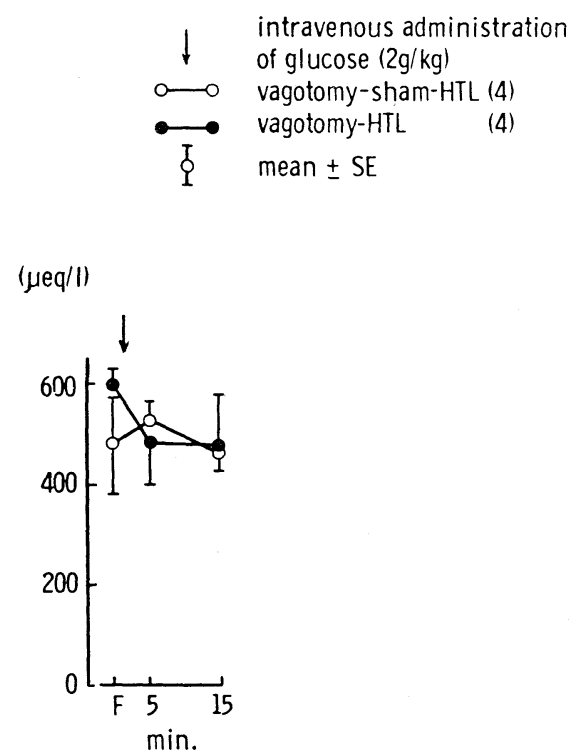

Fig. 4. Intravenous glucose tolerance tests in pair-fed vagotomized rats with or without hypothalamic lesions. Numbers in parentheses represent the number of rats. 
weeks after HTL is depicted in Fig. 4. In the vagotomy-HTL rats plasma IRI levels showed a more remarkable response than those in vagotomy-sham-HTL rats.

The relationships among plasma triglyceride levels, plasma IRI levels and grade of obesity

There was a significant correlation between plasma triglyceride levels and plasma IRI levels in vagotomized rats and their body weight, Lee's index and weight of adipose tissue, respectively (Table 2 ). The plasma IRI level was significantly correlated to the plasma triglyceride level in vagotomized rats with or without HTL (Fig. 5). Similar findings were obtained in rats pair-fed after vagotomy.
Table 2. Coefficients of correlation of plasma triglyceride or IRI levels with various parameters of obesity in vagotomized rats with or without hypothalamic lesions.

\begin{tabular}{|c|c|c|}
\hline & Plasma triglyceride & Plasma IRI \\
\hline Body weight (50) & $0.77^{*}$ & $0.69 *$ \\
\hline Lee's index (50) & $0.65^{*}$ & $0.54^{*}$ \\
\hline $\begin{array}{l}\text { Weight of epi- } \\
\text { didymal fat pad } \\
\text { (10) }\end{array}$ & $0.55^{* *}$ & $0.81^{* *}$ \\
\hline
\end{tabular}

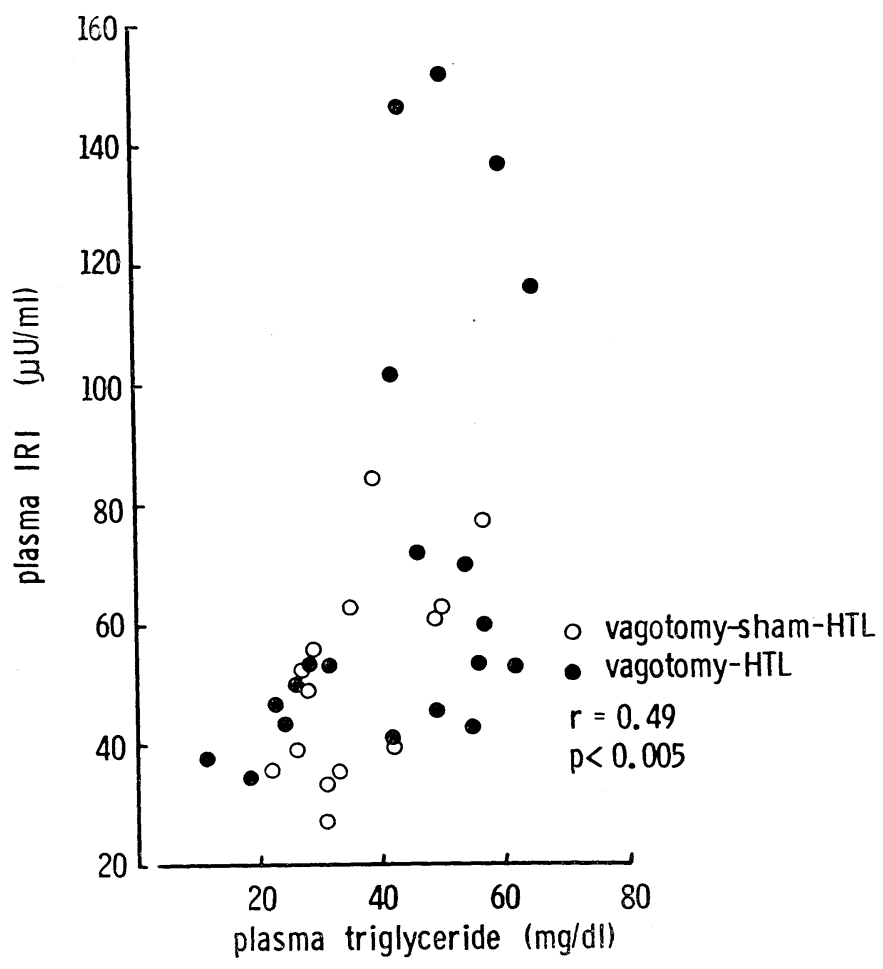

Fig. 5. Correlation between plasma IRI and triglyceride levels in vagotomized rats with or without hypothalamic lesions. 


\section{Discussion}

The occurrence of hyperinsulinemia in obese rats with HTL was described by Hales (1964) and others. We reported previously that the elevation of plasma IRI and enlargement of the pancreatic islets were found from the early stage after HTL and that increased secretion of insulin might play an important role in the development of obesity due to HTL (Chikamori el al., 1973).

Hales and Kennedy (1964) maintained that hyperinsulinemia in obese rats with HTL was attributable to hyperphagia. However, hyperinsulinemia is demonstrated in the HTL rats even when food intake is restricted (Suzuki, 1970). It is also seen in hypophysectomized rats with HTL (Hamajo, 1974) and in rats which had pituitary hormone replaced following HTL. Accordingly, it seems difficult that the cause of hyperinsulinemia is attributed only to hyperphagia or pituitary dysfunction.

The area of the ventromedial hypothalamus corresponds to the $b$-sympathetic zone designated by Kurotsu et al. (1953). Kuzuya (1962) found that electric stimulation of the lateral hypothalamic area (c-parasympathetic zone) induced an increased secretion of insulin. Kaneto et al. (1966) observed increased IRI secretion in the canine following the electric stimulation of the vagal nerve. These observations suggest that the rats with HTL become vagotonic, and that their insulin secretion is stimulated by the mediation of the vagal nerve, resulting in hyperinsulinemia.

In order to clarify such interactions, we performed serial experiments using HTL rats with truncal vagotomy. VagotomyHTL rats showed a smaller and more delayed increase in food intake and in body weight than sham-vagotomy-HTL rats. However, both food intake and body weight were greater in vagotomy-HTL rats than those in vagotomy-sham-HTL rats throughout the experiment. By pair-feeding after vagotomy, obesity due to HTL also developed.

Hyperinsulinemia in HTL rats was reduced in degree and delayed by vagotomy. In sham-vagotomy-HTL rats, significant hyperinsulinemia occurred 1 to 4 weeks after HTL, but in this period there was no significant difference in plasma IRI levels between vagotomy-HTL and vagotomysham-HTL rats, suggesting the effect of vagotomy. Fifteen weeks later, however, significant hyperinsulinemia was also recognized in vagotomy-HTL rats. Similar findings were obtained when food intake was kept constant by pair-feeding after vagotomy, and the IRI levels in the intravenous glucose tolerance test were also obviously elevated in HTL rats.

These results suggest that the increased insulin secretion under the control of hypothalamus-vagal nerve-pancreatic islets system may play an important role in the occurrence of hyperinsulinemia in hypothalamic obesity. On the other hand, factors other than vagal nerve should be taken into account, since hyperinsulinemia due to HTL is also observed in vagotomized rats, although its degree is less than in sham-vagotomy-HTL rats.

It has been reported that the lateral hypothalamic area contains a factor which stimulates insulin secretion (Idahl and Martin, 1971). On the other hand, somatostatin, recently isolated from the hypothalamus, was demonstrated to inhibit insulin secretion (Alberti et al., 1973). Therefore, it does not seem unlikely that some kind of humoral factors from the hypothalamus may participate in the development of hyperinsulinemia in rats with HTL.

As another pathogenetic factor in the hyperinsulinemia in this type of obesity, an increase in insulin antagonists may be 
considered. In recent years, to the importance of cellularity in the adipose tissue attention has been drawn in this point. As reported by Hirsch and Han (1969), the fat cell size is increased in obese humans or in obese rats. Salans et al. (1968) reported that the insulin action on glucose metabolism was reciprocally correlated with the cell size in adipose tissues. As we reported previously, plasma IRI level is significantly correlated with body weight, Lee's index or epididymal fat weight in HTL rats. Similar relationship could be demonstrated in the present experiment in vagotomized rats or pair-fed rats after vagotomy.

Increased plasma lipids may play the part of an insulin antagonist. We had reported that plasma FFA or triglyceride levels were elevated in HTL rats and there was a significant correlation between plasma IRI and plasma triglyceride levels (Chikamori et al. 1973). Similar findings were obtained in the present study in vagotomized rats or pair-fed rats after vagotomy. It may be possible that hyperinsulinemia in HTL rats is induced by obesity, or by a concomitant increase in adipose tissues and blood lipids.

Thus, it is suggested that the hyperinsulinemia in rats with HTL may be not only due to insulin hypersecretion from the pancreas mediated via vagal nerve system, but also due to some insulin-antagonistic factors as increases of adipose tissues and plasma lipids.

\section{Acknowledgement}

The authors are deeply grateful to Prof. H. Mori and Prof. S. Saito of Tokushima University for their kind advice.

\section{References}

Alberti, K. G. M. M., N. J. Christensen, S. E. Christensen, A. P. Hansen, J. Iversen, K. Lundaek, K. Seyer-Hansen and H. Ørskov (1973). Lancet 2, 1299.

Chikamori, K. (1969). Shikoku Acta Med. 25, 608. (In Japanese)

Chikamori, K., K. Masuda, H. Izumi and U. Tezuka (1973). Folia Endocrinol. Jap. 49, 344. (In Japanese)

Fletcher, M. J. (1968). Clin. Chim. Acta 22, 393.

Frohman, L. A. and L. L. Bernerdis (1971). J. Comp. Neur. 141, 107.

Hales, C. N. and G. C. Kennedy (1964). Biochem. J. 90, 620 .

Hamajo, T. (1974). Shikoku Acta Med. 30, 228. (In Japanese)

Hirsch, J. and P. W. Han (1969). J. Lipid Res. 10, 77.

Idahl, L. A. and J. M. Martin (1971). J. Endocr. 51,601 .

Itaya, K. and M. Ui (1965). J. Lipid Res. 6, 16.

Kaneto, A., K. Kosaka and K. Nakao (1966). Endocrinology 80, 530.

Kurotsu, T., C. Tabayashi and T. Ban (1953). $J$. Osaka Univ. 3, 529.

Kuwata, T. (1970). Shikoku Acta Med. 26, 524. (In Japanese)

Kuzuya, T. (1962). J. Jap. Soc. Intern. Med. 51, 1049. (In Japanese)

Lee, M. O. (1929). Am. J. Physiol. 89, 24.

Salans, L. B., J. L. Knittle and J. Hirsch (1968). J. Clin. Invest. 47, 153.

Snowdon, C. T. and A. N. Epstein (1970). J. Comp. Physiol. Psychol. 71, 59.

Suzuki, H. (1970). Shikoku Acta Med. 26, 574. (In Japanese) 